\title{
A fast neural signature of motivated attention to consumer goods separates the sexes
}

\author{
Markus Junghöfer ${ }^{1}$, Johanna Kissler ${ }^{2}$, HaraldT. Schupp ${ }^{3}$, Christian Putsche $^{1}$, Ludger Elling ${ }^{1}$ and Christian Dobel ${ }^{1}$ \\ Institute for Biomagnetism and Biosignalanalysis, University of Münster, Münster, Germany \\ 2 Department of Clinical Psychology, University of Konstanz, Konstanz, Germany \\ ${ }^{3}$ Department of General Psychology, University of Konstanz, Konstanz, Germany
}

\section{Edited by:}

Hauke R. Heekeren, Max Planck Institute for Human Development, Germany

\section{Reviewed by:}

Manfred Herrmann, University of Bremen, Germany

Annekathrin Schacht, Humboldt

University, Germany

Andreas Mühlberger, University of

Würzburg, Germany

${ }^{*}$ Correspondence:

Markus Junghöfer, Institute for

Biomagnetism and Biosignalanalysis,

University of Münster,

Malmedyweg 15, 48149 Münster,

Germany.

e-mail: markus.junghoefer@

uni-muenster.de
Emotional stimuli guide selective visual attention and receive enhanced processing. Previous event-related potential studies have identified an early (>120 ms) negative potential shift over occipito-temporal regions (early posterior negativity, EPN) presumed to indicate the facilitated processing of survival-relevant stimuli. The present study investigated whether this neural signature of motivated attention is also responsive to the intrinsic significance of man-made objects and consumer goods. To address this issue, we capitalized on gender differences towards specific man-made objects, shoes and motorcycles, for which the Statistical Yearbook 2005 of Germany's Federal Statistical Office (Statistisches Bundesamt, 2005) revealed pronounced differences in consumer behavior. In a passive viewing paradigm, male and female participants viewed pictures of motorcycles and shoes, while their magnetoencephalographic brain responses were measured. Source localization of the magnetic counterpart of the EPN (EPNm) revealed pronounced gender differences in picture processing. Specifically, between 130 and 180 ms, all female participants generated stronger activity in occipito-temporal regions when viewing shoes compared to motorcycles, while all men except one showed stronger activation for motorcycles than shoes. Thus, the EPNm allowed a sex-dimorphic classification of the processing of consumer goods. Self-report data confirmed gender differences in consumer behavior, which, however, were less distinct compared to the brain based measure. Considering the latency of the EPNm, the reflected automatic emotional network activity is most likely not yet affected by higher cognitive functions such as response strategies or social expectancy. Non-invasive functional neuroimaging measures of early brain activity may thus serve as objective measure for individual preferences towards consumer goods.

Keywords: emotion, attention, MEG, EEG, consumer goods, EPN, EPNm

\section{INTRODUCTION}

Emotional cues guide selective visual attention and receive enhanced processing (Lang et al., 1997; Öhman et al., 2000; Tucker et al., 2000; Vuilleumier, 2005). Functional magnetic resonance imaging (fMRI) revealed increased blood oxygen level dependent (BOLD) signals in associative visual regions (extrastriate, occipito-parietal, and inferior temporal cortex) and subcortical limbic structures when viewing emotionally arousing compared to neutral pictures (e.g., Bradley et al., 2003; Junghöfer et al., 2005; Sabatinelli et al., 2005). Event-related brain potential studies are particularly informative with regard to the temporal dynamics of emotion processing in the visual brain. Using naturalistic scenes from the international affective picture system (IAPS, Lang et al., 2005), a difference in processing emotional (pleasant and unpleasant) compared to neutral pictures is reflected by an enhanced negativity over temporooccipital sites (Junghöfer et al., 2001; Keil et al., 2002; Schupp et al., 2003; Wieser et al., 2006). This early posterior negativity (EPN) develops around $120 \mathrm{~ms}$ after stimulus onset and lasts until about $300 \mathrm{~ms}$. EPN modulation is most accentuated for pictures depicting evolutionary relevant stimulus contents such as reproduction and defense (Schupp et al., 2003), which also provoke reliable somatic, autonomic, and humoral responses (Bradley et al., 2001). Estimates of the generating sources for these differential ERP activities suggested that emotional stimuli receive enhanced processing in occipito-temporo-parietal regions, particularly pronounced for right-hemispheric regions (Junghöfer et al., 2001; Schupp et al., 2003). The measurement of event-related field potentials (derived from MEG) provides a complementary look at the spread of activation in the visual processing stream. The magnetic counterpart of the EPN consists of two distinct peaks of activation. Strongest effect sizes were observed for the first EPNm peak between 110 and $170 \mathrm{~ms}$ indicating the amplified processing of emotional pictures in occipito-parieto-temporal visual processing areas (Peyk et al., 2009). In combination, these findings have been considered from the perspective of "natural selective attention", proposing that stimulus perception and evaluation are in part directed by underlying motivational systems of avoidance and approach (Lang et al., 1998).

It is an enduring challenge to determine the sensitivity of the EPN component to distinct classes of emotional stimuli. There is accumulating evidence that the EPN component is not only modulated by comparatively high-arousing IAPS pictures but also by less intense emotional stimuli. Facial expression provides important information in social interaction and previous research revealed a larger EPN component to threatening and fearful as compared to neutral facial expressions (Schupp et al., 2004; Leppänen et al., 
2007; Mühlberger et al., 2009; Wieser et al., 2010). Further studies revealed the emotion-attention relationship with regard to symbolic signal systems, which demonstrated the power of ontogenetic experience and learning in directing selective attention during perceptual encoding. Specifically, emotional hand gestures of insult and approval and the reading of emotional (pleasant and unpleasant) adjectives compared to neutral control gestures and words led to increased EPN amplitudes (Kissler et al., 2007; Flaisch et al., 2009; Schacht and Sommer, 2009). Furthermore, a recent study revealed that attitude inconsistent moral statements also elicited increased EPN amplitudes (Van Berkum et al., 2009). These findings demonstrate the emotion-attention relationship indicated by the EPN component across biologically prepared and socially shared emotional signal systems.

From a conceptual perspective, EPN modulation has been investigated with respect to stimulus materials engaging an emotional associative network representing events and their associated consequences for the self and others, as well as approving or disapproving actions of agents. In their organizational scheme of emotion types, Ortony et al. (1988) suggested the liking and disliking of objects as a further principal class of emotions. Accordingly, the main aim of the present study was to investigate whether the EPN component is sensitive to differences in the liking of man-made objects. Addressing this issue seems also relevant to the field of neuroeconomics. Previous functional imaging studies revealed increased neural activations in reward sensitive and visual processing structures to attractive sport cars and logos of favorite brands (Erk et al., 2002; Schaefer and Rotte, 2007). Extending this research, the EPNm may prove to be useful to reveal individual differences towards consumer goods at the level of automatic perceptual encoding.

The present study investigated the emotion-attention relationship indicated by the EPN component with regard to man-made, cultural objects. For this purpose a between-group design was chosen, building on pronounced differences towards motorcycles and shoes between the sexes. Based on Germany's Statistical Yearbook for 2005 women spent on average $109 €$ for shoes, whereas men spent only about half of this amount $(61 €)$. In contrast, 4.5 times as many men $(10.8 \%)$ than women $(2.6 \%)$ owned motorcycles in that year. Assuming that consumer preferences reflect affective differences in cell ensembles representing these cultural objects, an interaction of gender by object category was predicted with stronger responses to motorcycles in men and stronger responses to shoes in women.

\section{MATERIALS AND METHODS PARTICIPANTS}

A total of 20 right-handed participants (10 males) with normal or corrected-to-normal vision ${ }^{1}$ took part in the experiment. All participants were students at the University of Münster and members of a subject pool for the Institute for Biomagnetism. They received a financial bonus of 9 Euros for participation. Male and female participants did not differ with respect to age (mean: 25 years), years of education (mean: 13) and the typical disposable personal income for a student in Germany (mean: 750 Euros per month).

${ }^{1}$ Purpose built glasses without any magnetic material were used for the study.
All subjects read and signed a consent form, informing about the nature of the study, which has been approved by the University of Münster Institutional Review Board.

\section{STIMULUS MATERIALS}

In total, 160 experimental stimuli were used, consisting of side views of 80 different motorbikes and 80 different single shoes (40 men's and 40 women's shoes). Images were taken from the Internet, as well as from several picture-databases. A sample of typical stimuli is shown in Figure 1. Stimuli were shown in random sequence, each stimulus being presented both in its original form and in a horizontally mirrored version to avoid effects from differing viewing angles. Therefore, two sets of pictures were created by horizontally mirroring all images. All pictures had a visual resolution of $60 \mathrm{pixel} /$ $\mathrm{cm}$, had an image size of $6 \times 6 \mathrm{~cm}$, and were presented on a white background at a viewing distance of $50 \mathrm{~cm}$ (6.9 visual angle).

\section{EXPERIMENTAL PROCEDURE}

Participants were seated in a dimly lit, sound-attenuated, and magnetically shielded chamber. Their head shape was digitized using a Polhemus 3 Space ${ }^{\circledR}$ Fasttrack prior to the measurement. Three landmark coils at the pre-auricular points and the nasion of the subject were determined in order to calculate their relative head position within the MEG scanner for later source analysis. Stimuli were projected onto a screen by means of a mirror system. The exact physical stimulus onset timing was measured using a photodiode 2 .

The experiment was divided into two experimental blocks with a short interim break in which subjects were allowed to do slight relaxation body and head movements. The first block presented all 160 stimuli with a pseudo-random orientation - left and right views appeared equally often within each picture category - the second block presented the corresponding 160 mirrored images. Stimulus category order was completely randomized within the blocks. The pictures were presented for $1 \mathrm{~s}$ replacing a small centered fixation cross. The fixation cross was shown throughout the inter-stimulusintervals, which lasted on average $2 \mathrm{~s}$ (range: 1.6-2.4 s).

At the beginning of the experiment subjects were informed that they were going to see different visual stimuli they should attend to. They were asked to focus their gaze on the fixation cross and avoid body movements during stimulation. No other instruction was given. After the MEG recording, participants completed a questionnaire encompassing items aimed at accessing their general attitudes towards and experience with shoes and motorcycles. Subjects were requested to list all shoe brands they know, and to specify how many shoes they own, how often they shop for shoes, and how much they spend for a new pair. Concerning motorcycles, participants had to list the different brands they know, indicate whether they read motorcycle magazines, and if they would like to own or buy a motorcycle. The complete questionnaire is given in the Appendix.

\footnotetext{
${ }^{2}$ Invisible on the subject screen a small black square was added at one upper edge of the fixation cross frame that was presented during the inter-stimulus-interval. A photodiode positioned at this point in the beam sent a trigger signal of stimulus onset to the data recording device leading to a maximal imprecision of one sampling point (here $1.7 \mathrm{~ms}$ ). Due to different clocks in the beamer and the stimulation computer, picture onset would otherwise have had a temporal imprecision in the range of one video frame (here $16 \mathrm{~ms}$ ).
} 

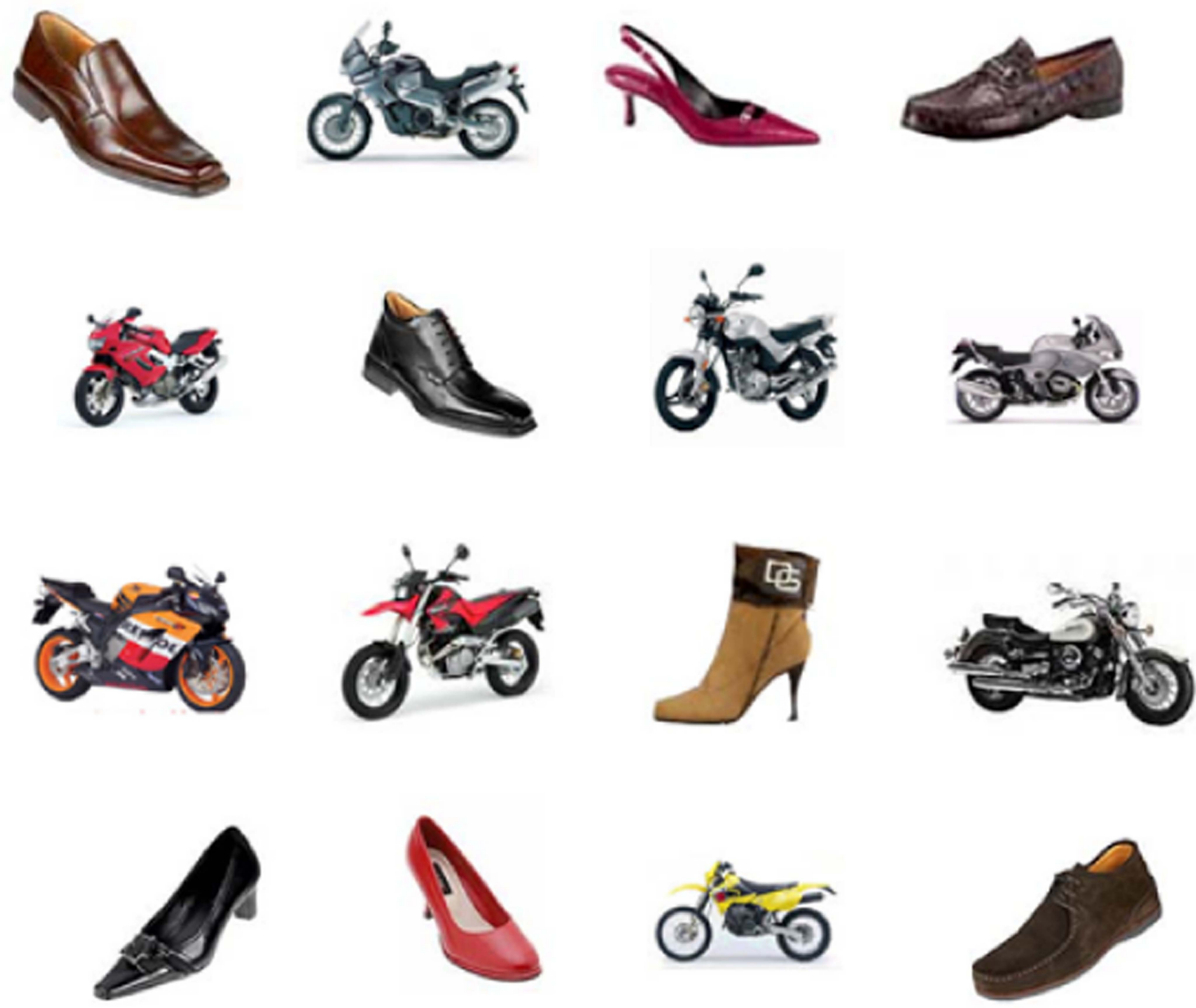

FIGURE 1| Examples from the $\mathbf{8 0}$ shoes (40 male shoes) and $\mathbf{8 0}$ motorcycles that were presented in pseudo-randomized fashion. Each stimulus was shown in left and right sided view.

Furthermore, participants also gave ratings of emotional arousal and hedonic valence for a subset of 20 representative shoes and 20 motorcycles, using a computerized version of the self assessment Manikin rating (Lang et al., 1998; nine-point scales).

\section{DATA ACQUISITION AND PRE-PROCESSING}

MEG signals were recorded using a 275-sensor whole-head MEGsystem (Omega 275, CTF, VSM MedTech Ltd.) with first-order axial SQUID gradiometers ( $2 \mathrm{~cm}$ diameter, $5 \mathrm{~cm}$ baseline, $2.2 \mathrm{~cm}$ average inter-sensor spacing). Data were recorded continuously with first-order gradient filtering. Responses were sampled at $600 \mathrm{~Hz}$ and filtered online with a frequency band-pass of $0-150 \mathrm{~Hz}$. The continuous data were then digitally high-pass filtered at $0.01 \mathrm{~Hz}$ and low-pass filtered at $48 \mathrm{~Hz}$ using zero-phase forward and reverse digital filters before stimulus synchronized epochs were extracted from $200 \mathrm{~ms}$ before stimulus onset to $600 \mathrm{~ms}$ after stimulus offset and baseline-adjusted for pre-stimulus (150 ms) activity.

Event-related fields were edited based on a method for statistical control of artifacts in high-density EEG/MEG measures (Junghöfer et al., 2000), which includes the extraction of globally noise-contaminated epochs and interpolation of regional contamination within trials. Average waveforms were calculated for the two experimental categories (motorcycles, shoes) for each sensor, experimental block, and participant. Testing for possibly differential effects for the comparison of motorcycles with male or female shoes, additionally separate average waveforms were calculated for both shoe categories. Adjusting the differing number of trials and thus signal to noise ratios for theses comparisons one half of the 160 motorcycle trials were randomly chosen and averaged.

\section{STATISTICAL ANALYSIS \\ Self-report data}

For each of the 10 motorcycle or 12 shoe items in the experience/expertise questionnaire subjects received between 0 and 12 points for motorcycle items and $0-10$ points for shoe items respectively, resulting in a maximal score of 120 points for each category. Repeated-measures ANOVAs with the factors gender and category were calculated for the corresponding questionnaire sum scores, as well as for the mean scores of the emotional arousal and hedonic valence ratings. In order to assess the probability of group/gender membership for each individual, we 
performed discriminant analyses based on the items of the questionnaire. First, we used the items of the questionnaire that showed group differences. Second, we entered the items for motorcycles and shoes separately into stepwise analysis procedures (inclusion criterion: $F=3.84$; exclusion: $F=2.71$ ), to learn whether each pool of items would be sufficient for group classification.

\section{Magnetoencephalographic data: source analysis}

Underlying cortical sourceswere estimatedbyapplyinga L2-minimumnorm estimate (L2-MNE; Hämäläinen and Ilmoniemi, 1994). The $\mathrm{MNE}$ is an inverse method allowing the reconstruction of distributed neural sources underlying the extracranially recorded event-related magnetic fields without the necessity of a priori assumptions regarding the number and possible locations of underlying neural generators. The L2-MNE is calculated by multiplying the pseudo-inverse of the so-called lead-field matrix (which describes the sensitivity of each sensor to the sources) with the averaged data.

Individual lead-field matrices were computed for each participant and experimental block, based on information about the center and radius of a sphere fitting best to the digitized head shape, and the locations and orientations of the MEG sensors relative to the head positioning in the MEG scanner. A spherical shell with 350 evenly distributed dipole locations served as the distributed source model. At each dipole location, two perpendicular and tangentially oriented dipoles were positioned. A shell radius of $87 \%$ of the individual head radius was chosen, roughly corresponding to the gray matter volume. L2-MNEs were computed for each experimental category, experimental block, and subject and then averaged across the two experimental blocks.

For the purpose of statistical analysis, source activity was analyzed using single-source waveform and conventional repeated-measures ANOVAs. In single-source waveform analyses, 2 (gender) $\times 2$ (category) ANOVAs were calculated for each time point after picture onset separately for each individual L2-MNE source. To control for spurious findings in the waveform analysis, significant effects were considered meaningful only when effects were observed for at least 10 ms (cf. Schupp et al., 2007). Results revealed significant interaction effects of gender $\times$ category in the time interval from 120 to $190 \mathrm{~ms}$. As illustrated in the statistical parametric maps of Figure 2A, gender $\times$ category interactions were maximal in the interval between 130 and $180 \mathrm{~ms}$, were localized in bilateral ventral secondary visual cortex areas, and suggested a right-hemispheric dominance.

For conventional ANOVA analysis, mean amplitudes from representative occipito-temporal source clusters were averaged from 130 to $180 \mathrm{~ms}$ (Figure 2B).

\section{Magnetoencephalographic data: sensor analysis}

In order to compare the current findings to ERF and ERP recordings obtained in previous studies, a MEG sensor standardization technique was applied to allow the calculation of grand

\section{A Gender x Category interaction in source space}

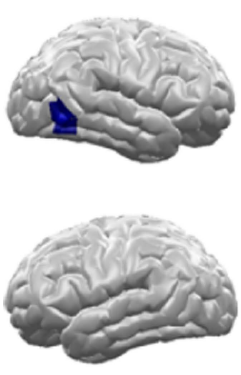

$120-130$
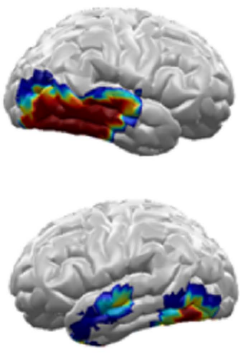

$130-140$
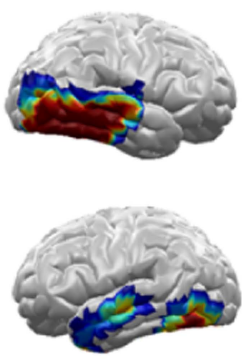

$140-170$
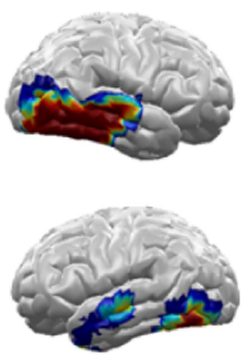

$170-180$
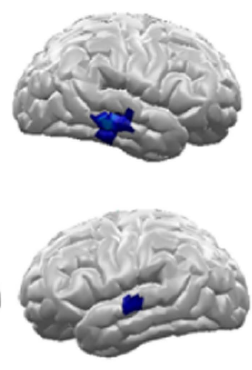

$180-190 \mathrm{~ms}$

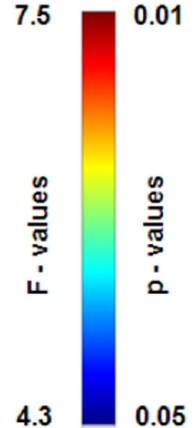

0.05

B

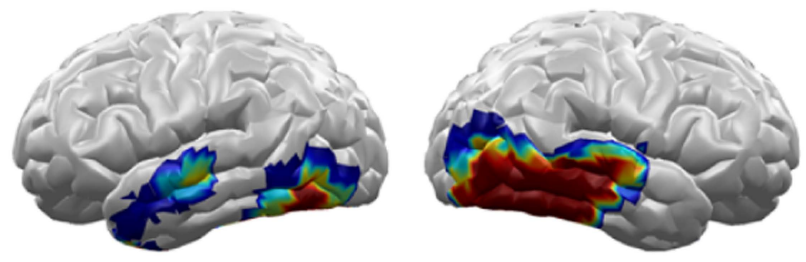

$130-180 \mathrm{~ms}$

FIGURE 2 | (A) Significant gender by category interactions of estimated neural activity (source space) were found in bilateral secondary areas of the ventral visual pathway between 120 and 190 ms after stimulus presentation - analyzed in 10 ms time bins. (B) Areas with significant gender by category interactions in the 130-180 ms time interval. 
mean MEG waveforms in the sensor space (cf. Junghöfer et al., 2006). Minimizing the variance due to differential positioning of the individual subjects head in the MEG scanner by a sensor standardization procedure, the event-related magnetic field for each participant was estimated as if were measured with a standardized sensor configuration identical for all participants in the scanner.

Similar to source space analysis, single sensor waveform analysis, and conventional ANOVA were conducted to explore the interaction of gender by category. Single sensor waveform analysis revealed significant gender by category interactions over right and left hemispheric occipito-temporo-parietal regions in a time interval from 110 to $230 \mathrm{~ms}$, most pronounced between 150 and $190 \mathrm{~ms}$ (see Figure 3A). For conventional ANOVA analysis, mean amplitudes were averaged across left and right occipito-temporo-parietal sensor regions (marked by cylinders in Figure 3B, right) in a time window from 150 to $190 \mathrm{~ms}$ and submitted to ANOVA analysis including factors of category, laterality, and gender.

For effects involving repeated measures, the GreenhouseGeisser procedure was used to correct for violations of sphericity.
Data pre-processing, inverse modeling,statistical analysis, and visualization were accomplished using ElectroMagneticEncephaloGraphy Software (Junghöfer and Peyk, 2004³).

\section{RESULTS}

\section{SELF-REPORT DATA}

The statistical analysis of the experience/expertise sum score revealed an interaction of gender and category $(F(1,18)=7.5$; $p<.05)$. Follow-up testing revealed a significant gender effect for motorcycle items $(t(18)=2.8 ; p<.05)$ with higher sum scores in males than females. In contrast, there was no gender difference for shoe items $(t(18)=-0.3)$. Furthermore, expertise sum scores revealed a significant main effect of category with more expertise for shoes than motorcycles across gender $(F(1,18)=5.67 ; p<.05)$

Item-based analysis of the motorcycle and shoe expertise items corroborated the findings of significant gender differences specifically for motorcycle items. Male subjects were more acquainted with different brands $(t(18)=5.2 ; p<.001)$ and types $(t(18)=5.5$; $p<.001)$ of motorcycles, they rode motorcycles $(t(18)=2.2 ; p<.05)$ and owned motorcycle drivers licenses more often $(t(18)=2.3$;

${ }^{3}$ www.emegs.org

\section{Gender x Category interaction in source space}

A

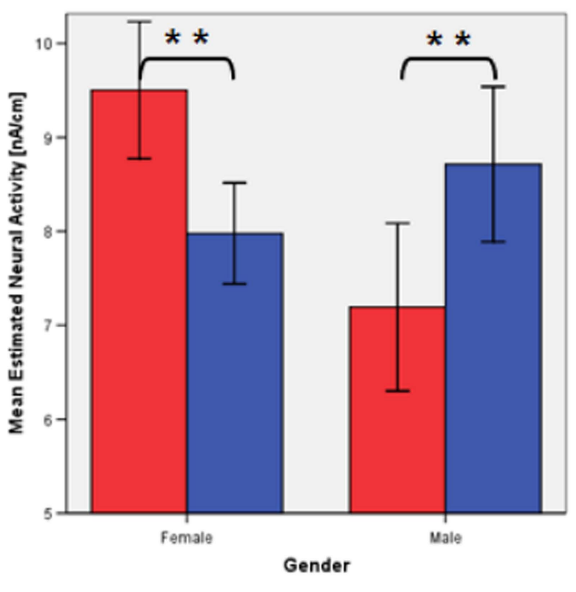

C
B

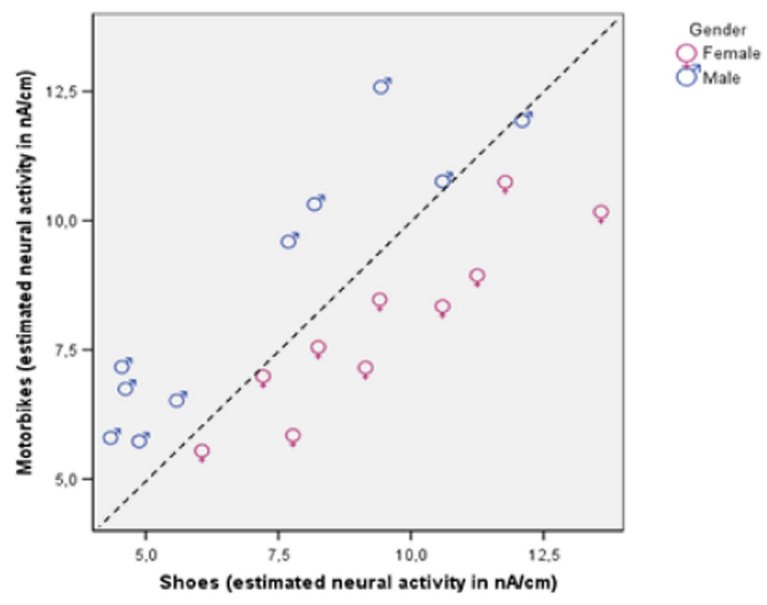

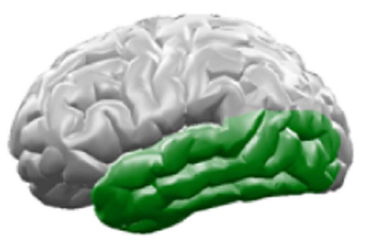

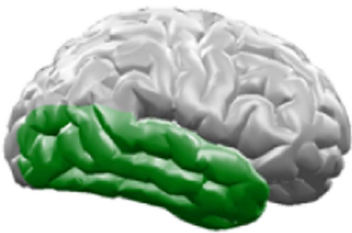

Regions of interest for

$130-180 \mathrm{~ms}$ time interval

FIGURE 3 | (A) Female subjects generated stronger neural activation when viewing shoes than when viewing motorcycles, whereas males revealed stronger activation for motorcycles than when viewing shoes. (B) All 10 female subjects showed stronger neural activities for shoes than for motorcycles, while nine of ten male subjects showed the opposite relation. (C) As marked in green, regions of interest for the analysis shown in (A,B) covered the occipito-temporal cortex except for primary visual areas. 
$p<.05)$ than female subjects. In contrast, male and female participants did not differ significantly in any of the 12 specific items for shoe expertise. There was only a trend for females visiting more shops than men $(t(18)=-1.99 ; p=.062)$ when shopping for shoes, but there was also a trend that men's sympathy for women was more influenced by women's "good shoe-taste" than vice versa $(t(18)=1.96 ; p=.065)$.

A discriminant analysis based on the six questionnaire items that had shown a statistical group difference, revealed that 95\% of participants (i.e., 19 of 20 subjects) were classified correctly. A similar result was received with a leave-one-out cross-validation analysis with $85 \%$ correctly classified participants (i.e., three subjects were misclassified). Entering only the items for motorcycles into a stepwise analysis revealed that only one variable was selected (number of known motorcycles) and that $85 \%$ of participants were correctly classified with this variable $(75 \%$ in the leave-one-out cross validation). Similarly, for the shoe-relevant items, $85 \%$ of participants were correctly classified $(75 \%$ in the leave-one-out cross validation) based on three variables (entering the analysis in the following order: number of visited stores for buying a pair of shoes, increased sympathy for good shoe-taste, amount of time spent on shoe care). Taken together, these results demonstrate that the randomly chosen groups of participants are compatible with the description of Germany's Federal Statistical Office (Statistical Yearbook 2005), i.e., that men when compared to women display a more positive attitude towards or higher expertise for motorcycles, while women show a slight tendency to care more about shoes.

The analysis of the arousal dimension revealed a main effect of category $(F(1,18)=7.8 ; p<.05)$, with motorcycles being more emotionally arousing than shoes for both sexes (mean arousal motorcycles/shoes - women: 5.0/4.1 - men: 4.3/3.0). The interaction of gender and category was not significant $(F(1,18)=0.2)$. Exploratory testing revealed that shoes approached significant gender differences $(t(18)=1.6 ; p=0.6$; one sided) with shoes being more arousing for women $($ mean $=4.1)$ than men $($ mean $=3.0)$. The analysis of the valence dimension revealed no significant main effects or interactions.

Finally, female shoes were rated as more arousing $(F(1,18)=40.5$; $p<.001)$ and received as trend higher valence ratings $(F(1,18)=3.4$; $p=.082)$ than male shoes but these rating differences were comparable for both genders $(F s<1)$.

\section{MAGNETOENCEPHALOGRAPHY: SOURCE ANALYSIS}

Beginning around $120 \mathrm{~ms}$ and ending around $190 \mathrm{~ms}$ after stimulus onset, highly significant interactions of gender and stimulus category within the ventral visual stream at secondary but not primary occipital and at temporal cortex areas were observed. No main effects of gender or stimulus category were found in this time interval and these areas. The statistical parametric maps for this interaction within the time intervals of interest are visualized in Figure 2A. As interactions were strongest in the interval between 130 and $180 \mathrm{~ms}$, the mean estimated neural activity within this interval was used for further calculation. Strongest effects were found around $165 \mathrm{~ms}$ in both hemispheres. Based on our hypothesis and the statistical parametric map for this time interval of interest (Figure 2B), regions of interest were defined for each hemisphere covering secondary occipital and the complete temporal cortex areas (Figure 3C).
The ANOVA with category and hemisphere as within factors and gender as between subject variable revealed a highly significant interaction of gender $\times$ category $(F(1,18)=43.3 ; p<0.0001)$ with stronger estimated neural activation for shoes compared to motorcycles in female and stronger activation for motorcycles compared to shoes in male subjects (Figure 3A). No main effects of category, hemisphere, and gender and no other interactions were significant $(F s(1,18)<1$; ns $)$. Separate ANOVAs for males and females revealed similarly strong main effects of stimulus category (males: $F(1,9)=20.3$; $p<0.001$; females: $F(1,9)=21.1$; $p<0.001)$ and again no effects of Hemisphere or category $\times$ hemisphere (all Fs $(1,18)<1$; ns). A scatter plot illustrating the estimated neural activities for each individual elucidates these strong effect sizes, as all 10 female subjects generated stronger neural activity when viewing shoes compared to motorcycles, while all men except one generated stronger activation for motorcycles than shoes (Figure 3B).

Correlations of estimated neural activity in each hemisphere and for each category with the questionnaire sum scores for shoes and motorcycles revealed a significant correlation for right-hemispheric activity evoked by motorcycles with the motorcycle sum score $(r(20)=0.40 p<.05$; one sided). Separate analyses for male and female participants showed that this effect was significant for men only $(r(10)=0.64 ; p<.05)$. Correlations with the shoe sum scores did not reach significance. However, testing a slightly later time interval between 150 and $200 \mathrm{~ms}$ resulted in a significant correlation between activity evoked by shoes with the shoe sum score $(r(20)=0.55 p<.01$; one sided $)$. This correlation was significant for females only $(r(10)=0.65 ; p<.05)$.

Separate ANOVAs with category factors "motorcycles vs. male shoes" and "motorcycles vs. female shoes" (adjusted trial number, see section Materials and methods) and hemisphere as within factors and gender as between subject variable revealed similar effects with highly significant gender by category interactions for the contrast "motorcycle vs. male shoes" $(F(1,18)=40.7$; $p<0.0001)$ and "motorcycle vs. female shoes" $(F(1,18)=19.8$; $p=0.001)$. Finally, comparing "female vs. male shoes" confirmed stronger activation for all shoes in female compared to male subjects $(F(1,18)=6.0 ; p=0.05)$, but revealed no interaction with gender $(F<1)$.

While Figure 2 gives the impression of stronger category by gender interaction effects over right occipito-temporal regions, with significant correlations between neural activity and questionnaire data limited to the right hemisphere, moving window analyses with a 10- or 20-ms step width did not support a category by hemisphere by gender interaction at any time point between 110 and $200 \mathrm{~ms}$.

\section{Magnetoencephalographic data: sensor analysis}

The analysis of event-related magnetic fields provided corroborating evidence for gender differences in processing shoes and motorcycles. Waveform analysis revealed a highly significant interaction of Category by Gender over left and right occipito-temporo-parietal sensor regions between 130 and 230 ms (Figure 4A).

Analyses of MEG sensor regions of interest (see Figure 4C) in the 150-190 ms time interval revealed a highly significant category by gender by hemisphere interaction $(F(1,18)=18.5 ; p<0.0001)$. The inspection of magnetic field maps revealed that right-hemispheric 
A
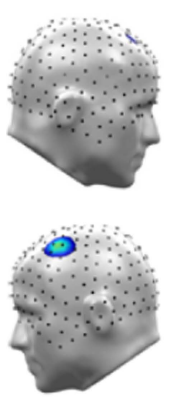

$110-130$ Gender x Category interaction in sensor space
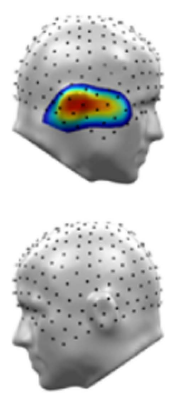

$130-150$
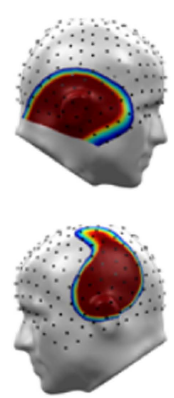

$150-170$
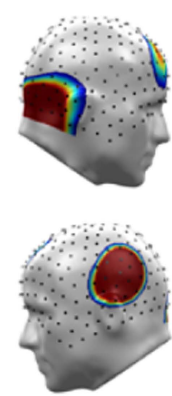

$170-190$
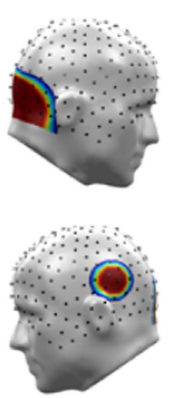

$190-210$
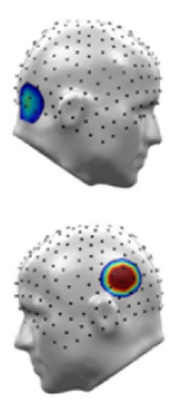

210-230ms

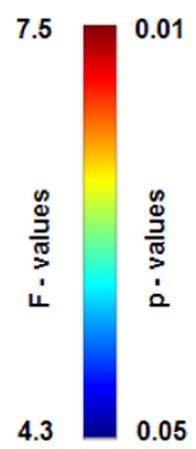

B Evoked magnetic fields in $150-190$ ms interval c

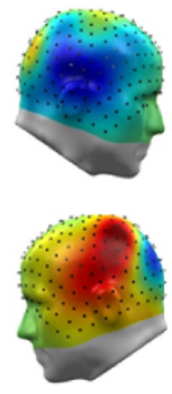

Femshoe
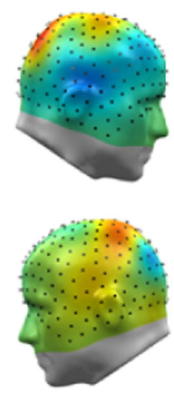

FemMoto
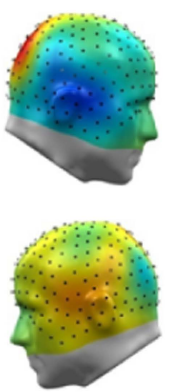

Maleshoe
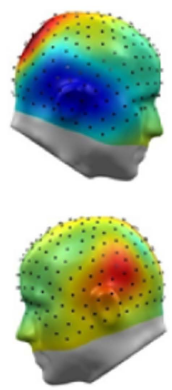

MaleMoto
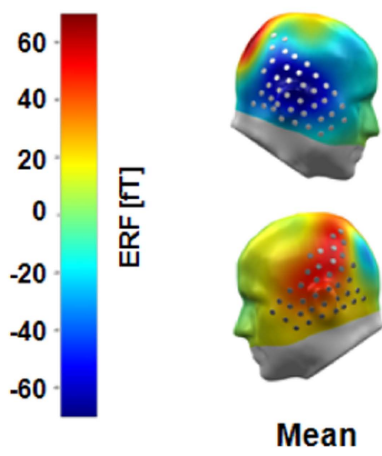

FIGURE 4 | (A) Significant gender by category interactions of evoked magnetic fields (sensor space) were found over left and right occipito-temporo-parietal sensor regions between 130 and $230 \mathrm{~ms}$ after stimulus presentation - analyzed in $20 \mathrm{~ms}$ time bins, MEG sensors projected on model head. (B) Means of event-related magnetic fields within a 150-190 ms time interval for each cell: stronger ingoing (negative) fields evoked by shoes compared to motorcycles in female subjects (FemShoe $>$ FemMoto) but stronger ingoing fields for motorcycles compared to shoes in male participants (MaleMoto > MaleShoe) in the right and mirrored effects in the left hemisphere. (C) Left- and righthemispheric sensor regions of interest (150-190 ms). occipito-temporo-parieto sensors showed stronger negative (ingoing) field amplitudes evoked by shoes compared to motorcycles in female subjects but stronger negative amplitudes for motorcycles compared to shoes in male participants (see Figure 4B). Convergent to the right-left polarity reversal of mid-latency visually evoked fields inverted effects were found at the mirrored left hemispheric sensors. Neither the main effect category nor the main effect gender was significant.

\section{DISCUSSION}

The main aim of the present study was to reveal the emotionattention relationship with regard to man-made objects. Consumer behavior, serving as proximal variable for consumer attitude and hedonic liking, indicates pronounced differences between the sexes for motorcycles and shoes. In this study, gender differences in consumer behavior were reflected by pronounced differences in how the brains of males and females responded to these object categories. In males, the EPNm component was significantly larger for motorcycles compared to shoes. In females, the reverse pattern emerged. These findings suggest that the EPN/EPNm may serve as sensitive indicator of the liking of consumer objects.

\section{CONSUMER OBJECTS: THE EMOTION-ATTENTION RELATIONSHIP}

Pictures are widely used to evoke emotions in humans. From an associative network perspective (Lang, 1979), the symbolic representation of the picture media needs to match reality to a degree sufficient to ignite emotional cell assemblies. The EPN component is elicited during the engagement of emotional cell assemblies, providing an indicator of selective visual attention to significant cues (Schupp et al., 2006). In previous research, emotional stimuli modulating the EPN component relate to emotion types associated with events and agents (Ortony et al., 1988). Specifically, scenes of erotica and threat represent events, which are evaluated according to their consequences for the self and others. Emotional facial expressions relate to actions of agents signaling approval or disapproval. Building upon these findings, the present study investigated picture media relating to a further, distinct type of emotion: Objects evoking evaluative liking and disliking responses. Results showed that corresponding EPN patterns in female and male participants reflected consumer differences regarding motorcycles and shoes. Accordingly, the EPN may serve as reliable indicator for the emotion-attention relationship across distinct types of emotion associated with events, agents, and objects. 
Determining neural correlates of individual preferences and the processing of cultural artifacts have been previously investigated in the field of neuroeconomics. This research suggests that liked objects may engage the reward circuitry (e.g., ventral striatum, orbitofrontal cortex) and lead to heightened visual attention (Erk et al., 2002; Schaefer and Rotte, 2007). Providing a high temporal resolution, MEG enables an assessment of early brain responses, which are presumed to reflect a default mechanism of affective stimulus evaluation. Previous research and current findings revealed that the EPN component shows several features of automatic processing such as occurring spontaneously, independent from stimulus novelty, and without intention to categorize the stimuli (Codispoti et al., 2006; Schupp et al., 2006, 2008; Kissler et al., 2009). Furthermore, considering the early onset of the observed effects, it is reasonable to assume that object processing within the first $200 \mathrm{~ms}$ mainly reflects implicit processes and is little affected by "conscious" reflection or the implementation of "on-line" processing strategies. Supporting this reasoning, research using masking and attentional blink paradigms indicate that neural correlates of "conscious" stimulus recognition appear considerably later ( $>200 \mathrm{~ms}$ ) than the current findings (Halgren and Marinkovic, 1995; Sergent et al., 2005; Del Cul et al., 2007). Overall, the EPN component has the potential to serve as a probe for individual preferences towards consumer objects at the level of automatic stimulus evaluation.

The importance of implicit processes for decision-making and the usage of implicit measures are increasingly stressed by social psychologists and other researchers interested in choice and decision-making (Strack and Deutsch, 2004; Dijksterhuis et al., 2008). For a long time a normatively based definition of rational and explicit choice behavior was favored (Evans and Over, 1996) supposing that human beings are construed as "rational animals" capable of recognizing the value or utility of their actions. However, a variety of findings indicate that human decisions appear irrational from a normative viewpoint, leading to a more subjective definition of choice behavior, i.e., that subjects' behaviors are "... in a way that is generally reliable and efficient for achieving one's goals" (Evans and Over, 1996). The shift in perspective towards a "subjective" viewpoint is grounded in an evolutionary perspective (Cosmides and Tooby, 1996), as evolution "... cares about whether people achieve their goals... and psychology - to some extent at least - reflects these priorities" (Dijksterhuis et al., 2008). Supporting this notion, an increasing number of studies demonstrate the importance of implicit processes for human decision and choice behavior (e.g., Galdi et al., 2008; Custers and Aarts, 2010). A measure of implicit preference such as the EPN, which reflects the level of automatic perceptual and evaluative stimulus processing without any need for an explicit task and response set, may complement behavioral measures, such as the implicit association test (Greenwald et al., 1998), in important ways.

\section{GENDER DIFFERENCES IN CULTURAL OBJECT PREFERENCES}

Object categories of shoes and motorcycles were selected because of well-established and pronounced differences in consumer behavior between the sexes (Germany's Statistical Yearbook, 2005). At first glance, gender differences towards cultural objects appear to reflect learning and mechanisms related to gender socialization, however, according to recent evidence, a biological predisposition appears also possible.
Developmental research indicates sex-dimorphic toy preferences in children. Specifically, girls usually play more with dolls and dressing-up items, and thus objects that afford opportunities for care giving (Eisenberg et al., 1982; Miller, 1987; Campbell et al., 2000), while boys prefer transportation toys and thus objects which can be used actively, move in space and promote a movement characterized by propulsion (O’Brien and Huston, 1985; Benenson et al., 1997). Thus, adult gender differences towards shoes and motorcycles may originate already during childhood.

Interestingly, sex differences in the playing behavior of children have been explained both in terms of socialization and biology (Servin et al., 1999). While there is general agreement that sex stereotype reinforcement strongly influences toy preferences (Ruble and Martin, 1998), there is also increasing evidence for a biological predisposition. For instance, gender specific toy preferences have already been found in 3- to 8-month-old infants (Alexander et al., 2009), and even in newborns (Connellan et al., 2000), and in two non-human primate species (Alexander and Hines, 2002; Hassett et al., 2008). Girls with congenital adrenal hyperplasia (CAH), a syndrome resulting in overproduction of adrenal androgens from early fetal life, are behaviorally masculinized and show stronger preferences for toys typically preferred by boys (Berenbaum and Hines, 1992; Hines and Kaufman, 1994). Importantly, this sextype toy playing behavior correlates with the degree of prenatal androgen exposure and appears rather independent of parental influence (Nordenström et al., 2002). The pathway leading from biological differences between the sexes to different preferences for consumer goods is complex and currently not known. One possibility is that higher levels of androgens available during a certain period of brain development affect visual processing pathways, and eventually result in gender differences that are reflected as preference or bias for perceptual features (Alexander, 2003). Of course, innate differences in androgens between the sexes may be augmented by social encouragement, enabling the interaction of nature and nurture (Hines and Alexander, 2008). Overall, it cannot be resolved whether the emotion-attention relationship towards motorcycles and shoes observed in the present study provides a "pure" case of socio-cultural learning or builds upon biological predisposition.

\section{STRENGTHS AND LIMITATIONS OF THE STUDY}

The design of this study allows examining the issue to what extent the EPN/EPNm reflects affective stimulus evaluation distinct from differences in low-level perceptual stimulus characteristics and object category. Perceptual differences in figure/ground composition and manipulations of image size affect the ERP wave and emotional arousal modulation in the EPN time window (De Cesarei and Codispoti, 2006; Bradley et al., 2007). Physical stimulus differences are excluded as potential confound in the present study, as the gender differences are based on the processing of the same objects. Furthermore, and particularly relevant for the study of man-made objects, hemodynamic studies have revealed an increasing object selectivity of higher level processing areas, possibly leading to pronounced differences as a function of stimulus category (Downing et al., 2001; Schwarzlose et al., 2005). The between-group design and the observed double dissociation, with a stronger response to shoes and motorcycles for women and men, respectively, provides 
direct evidence that amplified sensory processing within the EPN time interval is independent of specific physical features and category differences.

However, the selection of the stimulus categories also provides limitations for the study. The object categories may not only differ between gender in terms of preference but also in terms of expertise and exposure. This confound appears specifically with regard to motorcycles, for which men revealed greater expertise. However, expertise for shoes revealed no gender differences in the self-report data, while brain responses showed gender specific differentiation. Furthermore, expertise and affect are not independent, as familiar stimuli are liked more, and individual preference presumably leads to exposure and expertise (Zajonc, 1980). Accordingly, future studies investigating preferences for stimuli within a single category, i.e., candies or cars (cf. Erk et al., 2002), may provide more conclusive evidence concerning this issue.

The current study revealed pronounced gender differences in brain responses to shoes and motorcycles, which, however, were not paralleled by differences in emotional arousal and valence. This is an interesting finding, which needs to be followed up by future research. In previous research, emotional EPN differentiation was usually associated with differences in stimulus arousal (e.g., Junghöfer et al., 2001; Schupp et al., 2004; Kissler et al., 2007; Flaisch et al., 2009). However, considered from the perspective of the three-system approach (Lang 1979), verbal report, behavior, and physiological responses are partially independent, and the arousal dimension may correspondingly

\section{REFERENCES}

Alexander, G. M. (2003). An evolutionary perspective of sex-typed toy preferences: Pink, blue, and the brain. Arch. Sex Behav. 32, 7-14.

Alexander, G. M., and Hines, M. (2002). Sex differences in response to children's toys in nonhuman primates (Cercopithecus aethiops sabaeus). Evol. Hum. Behav. 23, 467-469.

Alexander, G. M., Wilcox, T., and Woods, R. (2009). Sex differences in infants' visual interest in toys. Arch. Sex Behav. 38, 427-433.

Benenson, J. F., Liroff, E. R., Pascal, S. J., and Cioppa, G. D. (1997). Propulsion: A behavioural expression of masculinity. Br. J. Dev. Psychol. 15, 37-50.

Berenbaum, S. A., and Hines, M. (1992). Early androgens are related to childhood sex-typed toy preferences. Psychol. Sci. 3, 203-206.

Bradley, M. M., Codispoti, M., Cuthbert, B. N., and Lang, P. J. (2001). Emotion and motivation I: Defensive and appetitive reactions in picture processing. Emotion 1, 276-298.

Bradley, M. M., Hamby, S., Löw, A., and Lang, P. J. (2007). Brain potentials in perception: picture complexity and emotional arousal. Psychophysiology 44, 364-373.

Bradley, M. M., Sabatinelli, D., Lang, P. J., Fitzsimmons, J. R., King, W., and Desai, P. (2003). Activation of the

not reflect a unitary phenomenon. In particular when looking at emotional states of reduced intensity, arousal may diminish activity in these systems in an unbalanced way. To further address this issue, it might be informative to determine whether a reliable physiological measure of arousal, such as skin conductance response, would reveal a pattern of modulation corresponding to the EPNm.

\section{CONCLUSION}

The present results indicate that motivationally relevant stimuli, whether of a rather predisposed and innate nature, culturally acquired, or reflecting personal learning history and attitudes, guide selective visual attention as reflected by the EPN/EPNm. This early brain mechanism might have evolved in our ancestors to secure and enhance the chances of survival. In modern humans it might be a crucial factor influencing complex decision-making such as buying items of intrinsic value. As such, the measurement of fast neurophysiological responses commends itself as a reliable and objective measure to investigate implicit motivated attention to various object classes in distinct target groups.

\section{ACKNOWLEDGMENTS}

We would like to thank Lena Geiger, Isabella Paul, Thomas Elbert, Dirk Vorberg, Pienie Zwitserlood and Gregory A. Miller for their valuable contributions. This work was supported by the German Research Foundation (DFG); Grants SFB-TRR58-C1 and JU-445 5-1.

Leeuwen, M. L., and van der Leij, A. (2008). The Rational Unconscious: Conscious Versus Unconscious Thought in Complex Consumer Choice. UK: Social Psychology of Consumer Behavior; Psychology Press.

Downing, P. E., Jiang, Y., Shuman, M., and Kanwisher, N. (2001). A cortical area selective for visual processing of the human body. Science 293, 2470-2473.

Eisenberg, N., Murray, E., and Hite, T. (1982). Children's reasoning regarding sex-typed toy choices. Child Dev. 53, 81-86.

Erk, S., Spitzer, M., Wunderlich, A. P., Galley, L., and Walter, H. (2002). Cultural objects modulate reward circuitry. Neuroreport 13, 2499-2503.

Evans, J. S. B. T., and Over, D. E. (1996). Rationality and Reasoning. New York: Psychology Press.

Flaisch, T., Schupp, H., Renner, B., and Junghöfer, M. (2009). Neural systems of visual attention responding to emotional gestures. NeuroImage 45, 1339-1346.

Galdi, S., Arcuri, L., and Gawronski, B. (2008). Automatic mental associations predict future choices of undecided decision-makers. Science 321, 1046-1047.

Greenwald, A. G., McGhee, D. E., and Schwartz, J. L. K. (1998). Measuring individual differences in implicit cognition: The implicit association test. J. Pers. Soc. Psychol. 74, 1464-1480.

Halgren, E., and Marinkovic, K. (1995). "Neurophysiological networks integrating human emotions," in The Cognitive Neurosciences, ed. M. Gazzaniga (Cambridge, MA: MIT Press), 1137-1151.

Hämäläinen, M., and Ilmoniemi, R. J. (1994). Interpreting magnetic fields of the brain: minimum norm estimates. Med. Biol. Eng. Comput. 32, 35-42.

Hassett, J. M., Siebert, E. R., and Wallen, K. (2008). Sex differences in rhesus monkey toy preferences parallel those of children. Horm. Behav. 54, 359-364.

Hines, M., and Alexander, G. M. (2008). Monkeys, girls, boys and toys: A confirmation letter regarding "Sex differences in toy preferences: Striking parallels between monkeys and humans". Horm. Behav. 54, 478-479.

Hines, M., and Kaufman, F. R. (1994) Androgen and the development of human sex-typical behavior: Roughand-tumble play and sex of preferred playmates in children with congenital adrenal hyperplasia (CAH). Child Dev. 65, 1042-1053.

Junghöfer, M., Bradley, M. M., Elbert, T. R., and Lang, P. J. (2001). Fleeting images: A new look at early emotion discrimination. Psychophysiology 38, 175-178. 
Junghöfer, M., Elbert, T., Tucker, D. M., and Rockstroh, B. (2000). Statistical control of artifacts in dense array EEG/MEG studies. Psychophysiology 37, 175-178.

Junghöfer, M., and Peyk, P. (2004). Analysis of electrical potentials and magnetic fields of the brain. Matlab Select 2, 24-28. EMEGS software is freely available at http://www.emegs. org.

Junghöfer, M., Peyk, P., Flaisch, T., and Schupp, H. (2006). Neuroimaging methods in affective neuroscience: Selected methodological issues. Prog. Brain Res. 156, 123-143.

Junghöfer, M., Schupp, H., Stark, R., and Vaitl, D. (2005). Neuroimaging of emotion: Empirical effects of proportional global signal scaling in fMRI data analysis. NeuroImage 25, 520-526.

Keil, A., Bradley, M. M., Hauk, O., Rockstroh, B., Elbert, T., and Lang, P. J. (2002). Large-scale neural correlates of affective picture processing. Psychophysiology 39, 641-649.

Kissler, J., Herbert, C., Peyk, P., and Junghöfer, M. (2007). 'Buzzwords' - spontaneous cortical responses to emotional words. Psychol. Sci. 18, 475-480.

Kissler, J., Herbert, C., Winkler, I., and Junghöfer, M. (2009). Emotion and attention in visual word processing: An ERP study. Biol. Psychol. 80, 75-83.

Lang, P. J. (1979). A bio-informational theory of emotional imagery. Psychophysiology 16, 495-512.

Lang, P. J., Bradley, M. M., and Cuthbert, B. N. (1997). "Motivated attention: Affect, activation, and action," in Attention and Emotion: Sensory and Motivational Processes, eds P.J.Lang, R. F. Simons, and M. Balaban (Mahwah, NJ: Erlbaum), 97-135.

Lang, P. J., Bradley, M. M., and Cuthbert, B.N.(1998). Emotion and motivation: Measuring affective perception. J. Clin. Neurophysiol. 15, 397-408.

Lang, P. J., Bradley, M. M., and Cuthbert, B. N. (2005). International affective picture system (IAPS): Digitized photographs, instruction manual and affective ratings. Technical Report A-6. Gainesville, FL: University of Florida.

Leppänen, J.M., Kauppinen, P. K., Peltola, M. J., and Hietanen, J. K. (2007).
Differential electrocortical responses to increasing intensities of fearful and happy emotional expressions. Brain Res. 1166, 103-109.

Miller, C. L. (1987). Qualitative differences among gender-stereotyped toys: Implications for cognitive and social development. Sex Roles 16, 473-487.

Mühlberger, A., Wieser, M. J., Herrmann, M. J., Weyers, P., Tröger, C., and Pauli, P. (2009). Early cortical processing of natural and artificial emotional faces differs between lower and higher social anxious persons. J. Neural Transm. 116, 735-746.

Nordenström, A., Servin, A., Bohlin, G., Larsson, A., and Wedell, A. (2002). Sex-typed toy play behavior correlates with the degree of prenatal androgen exposure assessed by CYP21 genotype in girls with congenital adrenal hyperplasia. J. Clin. Endocrinol. Metab. 87, 5119-5124.

O'Brien, M., and Huston, A. C. (1985). Development of sex-typed play behavior in toddlers. Dev. Psychol. 21, 866-871.

Öhman, A., Flykt, A., and Lundqvist, D. (2000). "Unconscious emotion: evolutionary perspectives, psychophysiological data and neuropsychological mechanisms" in Cognitive Neuroscience of Emotion, eds R. D. Lane and L. Nadel (Oxford: Oxford University Press), 296-327.

Ortony, A., Clore, G. L., and Collins, A. (1988). The Cognitive Structure of Emotions. Cambridge: Cambridge University Press.

Peyk, P., Schupp, H., Elbert, T. R., and Junghöfer, M. (2009). Emotion processing in the visual brain: A MEG analysis. Brain Topogr. 20, 205-215.

Ruble, D. N., and Martin, C. L. (1998). "Gender development," In Handbook of Child Psychology: Vol. 3. Social, Emotional, and Personality Development, 5th Edn. W. Damon (series ed.) and N. Eisenberg (vol.ed.), (New York: Wiley), 933-1016.

Sabatinelli, D., Bradley, M. M., Fitzsimmons, J. R., and Lang, P. J. (2005). Parallel amygdala and inferotemporal activation reflect emotional intensity and fear relevance. Neuroimage 24, 1265-1270.
Sergent, C., Baillet, S., and Dehaene, S. (2005). Timing of the brain events underlying access to consciousness during the attentional blink. Nat. Neurosci. 8, 1391-1400.

Servin, A., Bohlin, G., and Berlin, L. (1999). Sex differences in 1-, 3-, and 5 -year-olds' toy-choice in a structured play-session. Scand. J. Psychol. 40, 43-48.

Schacht,A., and Sommer, W. (2009). Time course and task dependence of emotion effects in word processing. Cogn. Affect Behav. Neurosci. 9, 28-43.

Schaefer, M., and Rotte, M. (2007). Favorite brands as cultural objects modulate reward circuit. Neuroreport 18, 141-145.

Schupp, H. T., Flaisch, T., Stockburger, J., and Junghöfer, M. (2006). Emotion and attention: Event-related brain potential studies. Prog. Brain Res. 156, 31-51.

Schupp, H. T., Junghöfer, M., Weike, A. I., and Hamm, A. O. (2003). Attention and emotion: an ERP analysis of facilitated emotional stimulus processing. Neuroreport 14, 1107-1110.

Schupp, H. T., Öhman, A., Junghöfer, M., Weike, A. I., Stockburger, J., and Hamm, A. O. (2004). The facilitated processing of threatening faces: An ERP analysis. Emotion 4, 189-200.

Schupp, H. T., Stockburger, J., Bublatzky, F., Junghöfer, M., Weike, A. I., and Hamm, A. O. (2008). The selective processing of emotional visual stimuli while detecting auditory targets: An ERP analysis. Brain Res. 1230, 168-176.

Schupp, H. T., Stockburger, J., Codispoti, M., Junghöfer, M., Weike, A. I., and Hamm, A. O. (2007). Selective visual attention to emotion. J. Neurosci. 27, 1082-1089.

Schwarzlose, R. F., Baker, C. I., and Kanwisher, N. (2005). Separate face and body selectivity on the fusiform gyrus. J. Neurosci. 25, 11055-11059.

Statistisches Bundesamt (2005). Statistisches Jahrbuch 2005 für die Bundesrepublik Deutschland. Wiesbaden: Statistisches Bundesamt.

Strack, F., and Deutsch, R. (2004) Reflective and impulsive determinants of social behavior. Pers. Soc. Psychol. Rev. 8, 220-247.
Tucker, D. M., Derryberry, D., Luu, P., and Phan, K. L. (2000). "Anatomy and physiology of human emotion: Vertical integration of brainstem, limbic, and cortical systems," in The Neuropsychology of Emotion, ed. J. C. Borod (New York, NY: Oxford University Press), 56-79.

Van Berkum, J. J., Holleman, B., Nieuwland, M., Otten, M., and Murre, J. (2009). Right or wrong? The brain's fast response to morally objectionable statements. Psychol. Sci. 20, 1092-1099.

Vuilleumier,P.(2005).How brains beware: neural mechanisms of emotional attention. Trends Cogn. Sci. (Regul. Ed.) 9, 585-594.

Wieser, M. J., Mühlberger, A., KenntnerMabiala, R., and Pauli, P. (2006). Is emotion processing affected by advancing age? An event-related brain potential study. Brain Res. 96, 138-147.

Wieser, M. J., Pauli, P., Reicherts, P., and Mühlberger, A. (2010). Don't look at me in anger!? Enhanced processing of angry faces in anticipation of public speaking. Psychophysiology 47, 271-280.

Zajonc, R. B. (1980). Feeling and thinking: Preferences need no inferences. Am. Psychol. 35, 151-175.

Conflict of Interest Statement: The authors declare that the research was conducted in the absence of any commercial or financial relationships that could be construed as a potential conflict of interest.

Received: 04 March 2010; paper pending published: 06 May 2010; accepted: 29 August 2010; published online: 20 September 2010.

Citation: Junghöfer M, Kissler J, Schupp HT, Putsche C, Elling L and Dobel C (2010). A fast neural signature of motivated attention to consumer goods separates the sexes. Front. Hum. Neurosci. 4:179. doi: 10.3389/ fnhum.2010.00179

Copyright (c) 2010 Junghöfer, Kissler, Schupp, Putsche, Elling and Dobel. This is an open-access article subject to an exclusive license agreement between the authors and the Frontiers Research Foundation, which permits unrestricted use, distribution, and reproduction in any medium, provided the original authors and source are credited. 


\section{APPENDIX \\ MOTORCYCLES}

1) Please name all motorcycle brands you know.

2) Please name all motorcycle types you know.

3) Do you own or did you ever own a motorcycle? (yes/no)

4) Did you ever ride a motorcycle? (yes/no)

If not, would you like to once ride a motorcycle? (no/a bit/ yes/definitely)

5) Do you have a driver's license for motorcycles? (yes/no)

6) Do you own a motorcycle helmet or other motorcycle equipment? (yes/no)

7) Do you buy motorcycle magazines or watch motorcycle races? (never/rarely/sometimes/regularly)

8) Suppose you had $5.000 €$ at hand. What's the probability that you would use the money in order to buy a motorcycle $(0 \%$; $25 \% ; 50 \% ; 75 \% ; 100 \%$ ).

9) How much money would you be willing to spend for the maintenance of a motorcycle you got as a gift? (nothing $/<25$ $€ /<50 € /<75 € / 100 €$ and more)

10) Please rank the following five vacation types with 1 as most and 5 as least preferential.

(Hiking-tour/beach-vacation/city-tour/motorcycle-tour/ sailing-trip)

\section{SHOES}

1) Please name all shoe brands you know (no sports shoes):

2) How many pairs of shoes do you own and wear at least once a year?

( $1-4 ; 5-8 ; 9-12 ; 13-16$; more than 16 pairs $)$
3) How many pairs of shoes do you buy per year? $(0 / 1 ; 2 / 3 ; 4 / 5$; $6 / 7$; more than 7 pairs)

4) How much money do you typically spend for a pair of shoes? $(<50 € ; 50-100 € ; 100-150 € ; 150-200 € ;$ more than $200 €)$

5) Buying shoes, how many shoe shops do you visit? $(1 ; 2 ; 3 ; 4$; more than 4)

6) Buying shoes, how much time do you need to buy one pair of shoes?

(<30 min; 30-60 min; 60-90 min; 90-120 min; more than $120 \mathrm{~min})$

7) How often do you use shoe care products? (never/rarely/ sometimes/often)

8) Do you use shoetrees? (never/rarely/sometimes/often)

9) Do you wear specific shoes for specific events? (never/rarely/ sometimes/often)

10) Do you care that your shoes fit with your clothes? (never/ rarely/sometimes/often)

11) Do you care about your partner's/the other sexes'/shoe taste and shoe choices? (never/rarely/sometimes/often)

12) Does the shoe taste of others influence your sympathy for her/him?

(never/rarely/sometimes/often) 